\section{Staubsaugen für die Milbenforschung}

U m ein Gesamtbild der in Deutschland verbreiteten Hausstaubmilben zu erstellen, hat Novartis gemeinsam mit seinem spanischen Partner Leti als erstes Pharmaunternehmen eigene Forschungsprojekte durchgeführt.

Für die erste Studie - Allergy and Mites in Germany Assessment (AMIGA) - verteilten die Außendienstmitarbeiter von Novartis pro Saison an durchschnittlich 45 Ärzte je zwölf Probensets. Jedes dieser Sets enthielt ein Röhrchen mit einem Filter und einen Adapter, um das Röhrchen an einen Staubsauger anschließen zu können. Die Ärzte verteilten die Sets an Patienten, Bekannte und Mitarbeiter ihrer Praxis und baten sie, die Matratze ihres Bettes auf höchster Leistungsstufe an vier Stellen jeweils eine halbe Minute zu saugen. Insgesamt wurden rund 2.250 Proben ausgewertet, 550 bis 650 pro Saison.

Es zeigte sich, dass die amerikanische Hausstaubmilbe Dermatophagoides farinae in Deutschland häufiger vertreten ist als erwartet. Sie fand sich in $57,4 \%$ aller ausgewerteten Staubproben, die europäische Hausstaubmilbe Dermatophagoides pteronyssinus dagegen nur in $45,3 \%$ der Proben. Die Populationsdichte, also die Menge der pro Probe nachgewiesenen Milben, war jedoch für $D$. pteronyssinus mit durchschnittlich 943 Milben/g Staub höher als für $D$. farinae mit durchschnittlich 700 Milben/g Staub. Die Populationsdichte ist eine wichtige Determinante: Bei Werten von mehr als 100 Hausstaubmilben pro Gramm Staub ist von einem erhöhten Risiko für eine Sensibilisierung und eine bronchiale Hyperreagibilität auszugehen, bei Werten von über 500 Hausstaubmilben pro Gramm

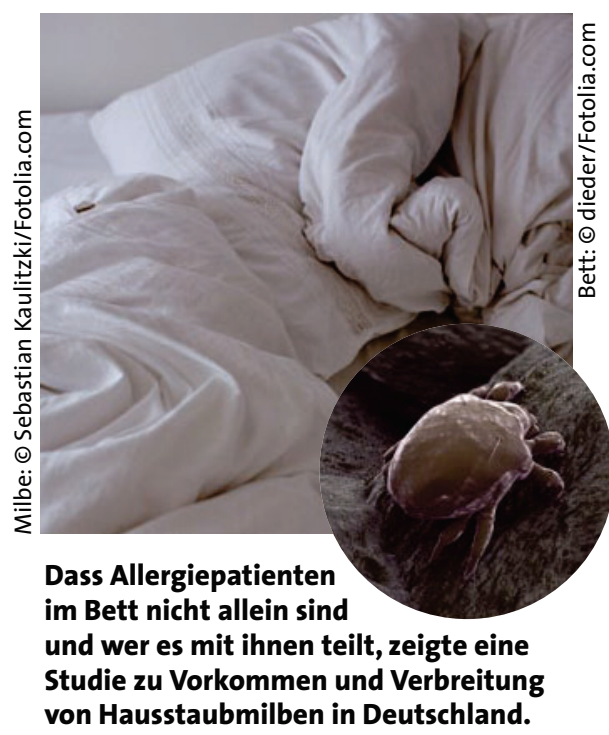

Staub von einem Anstieg des Risikoniveaus und einem erhöhtem Risiko für akute Asthmaanfälle. Die Auswertung bezogen auf die Jahreszeiten ergab hohe Populationsdichten der Milben in den Sommermonaten.

„Die Daten der AMIGA-Studie zeigen, dass bei der Allergendiagnostik grundsätzlich auf Hausstaubmilben getestet werden sollte, also auch bei Vorliegen einer saisonalen Allergie", lautete das Fazit von Dr. Guido Mühlmeier, Ulm. „Denn die Häufigkeit von Mischformen, also einer Pollen- und einer Hausstaubmilbenallergie, wird vielfach unterschätzt." Insgesamt spiegelt die Häufigkeit von Hausstaubmilben die Bedeutung der spezifischen Immuntherapie wider. Denn bei etwa $40 \%$ der Milbenallergiker müsse, wie Mühlmeier ausführte, mit einem Etagenwechsel gerechnet werden. Das häufige Auftreten von $D$. farinae und $D$. pteronyssinus ist für ihn - neben der bekannten hohen Kreuzallergenität beider Milbenarten - ein Argument, die spezifische Immuntherapie mit einem Kombinationspräparat zu bevorzugen, z. B. mit Depigoid ${ }^{\circledR}$ MilbenMix. Das Milbenallergoid ist zugelassen und erfüllt die strengen Anforderungen der World Allergy Organization. red

Nach Informationen von Novartis Pharma, Nürnberg

\title{
Physikalische Barriere gegen Allergene
}

D as pflanzliche Medizinprodukt Allergopret $^{\circledR}$ protect, ein Puder-Spray aus pflanzlicher Zellulose, wirkt rein physikalisch, verhindert das Auslösen der allergischen Reaktion und verringert nachweislich den Einsatz von Antihistaminika während der Allergiesaison. Bei leichteren allergischen Beschwerden, z.B. an Tagen mit wenig Pollenflug, kann bereits die alleinige Anwendung von Allergopret ${ }^{\circledR}$ protect ausreichend sein und chemischsynthetische Antiallergika ersetzen.

Das Wirkprinzip von Allergopret ${ }^{\circledR}$ protect ist einfach und effektiv: Nach dem Einsprühen in die Nase bildet sich innerhalb kurzer Zeit ein dünner Schutzfilm auf der Nasenschleimhaut, da das feuchte Milieu in der Nase die Zellulosepartikel zu einem Gel aufquellen lässt. Die so gebildete physikalische Barriere verhindert den direkten Kontakt von luftgetragenen Allergenen mit den sensibilisierten Mastzellen der Nasenschleimhaut. Ein Vorteil dieses Wirkprinzips: Als inerte Verbindung greift Zellulose nicht in den Stoffwechsel ein und macht nicht müde. Hierin unterscheidet das pflanzliche Puder-Spray sich von den meisten chemisch-synthetischen Mitteln wie etwa Antihistaminika. Allergopret ${ }^{\circledR}$ protect kann unbedenklich mit anderen Antiallergika kombiniert werden.

Dass der Einsatz des Zellulosefilms die gleichzeitige Verwendung von Anti- histaminika reduzierte, zeigte eine doppelblinde und plazebokontrollierte Studie von Jean Emberlin und Richard Lewis (Current Medical Research and Opinion 2006; 22: 275-85). Daran nahmen 97 Erwachsene mit saisonaler allergischer Rhinitis durch Pollen teil, die in den letzten zwei Jahren während der Heuschnupfenzeit Symptome gezeigt hatten.

Allergopret $^{\circledR}$ protect hilft bei Pollen-, Hausstaub- und Tierhaarallergie, ist sehr gut verträglich und schon für Kinder $\mathrm{ab}$ sechs Jahren geeignet. Es kann auch über einen längeren Zeitraum hinweg angewendet werden, macht nicht müde und trocknet die Nase nicht aus.

Gabriele Zörrgiebel

Nach Informationen von Bionorica SE, Neumarkt 